\title{
Generic Spin Model for the Honeycomb Iridates beyond the Kitaev Limit
}

\author{
Jeffrey G. Rau, ${ }^{1}$ Eric Kin-Ho Lee, ${ }^{1}$ and Hae-Young Kee ${ }^{1,2, *}$ \\ ${ }^{1}$ Department of Physics, University of Toronto, Toronto, Ontario M5S 1A7, Canada \\ ${ }^{2}$ Canadian Institute for Advanced Research/Quantum Materials Program, Toronto, Ontario MSG 1Z8, Canada
}

(Dated: March 18, 2014)

\begin{abstract}
Recently, realizations of Kitaev physics have been sought in the $\mathrm{A}_{2} \mathrm{IrO}_{3}$ family of honeycomb iridates, originating from oxygen-mediated exchange through edge-shared octahedra. However, for the $j_{\text {eff }}=1 / 2$ Mott insulator in these materials exchange from direct $d$-orbital overlap is relevant, and it was proposed that a Heisenberg term should be added to the Kitaev model. Here we provide the generic nearest-neighbour spin Hamiltonian when both oxygen-mediated and direct overlap are present, containing a bond-dependent off-diagonal exchange in addition to Heisenberg and Kitaev terms. We analyze this complete model using a combination of classical techniques and exact diagonalization. Near the Kitaev limit, we find new magnetic phases, $120^{\circ}$ and incommensurate spiral order, as well as extended regions of zigzag and stripy order. Possible applications to $\mathrm{Na}_{2} \mathrm{IrO}_{3}$ and $\mathrm{Li}_{2} \mathrm{IrO}_{3}$ are discussed.
\end{abstract}

The honeycomb family of iridium oxides[1-11] has attracted a considerable amount of attention [12-20] due to the possibility they lie near a realization of Kitaev's exactly solvable spin-1/2 honeycomb model[21]. This model hosts a number of remarkable features: a $Z_{2}$ spin liquid with gapless Majorana fermions and (non-Abelian) anyonic excitations under an applied magnetic field. No symmetry principle excludes terms besides the Kitaev, so additional interactions are generically expected. From microscopic calculations of exchange mediated through the edge-shared oxygen octahedra, it has been proposed that a pure Kitaev model of $j_{\mathrm{eff}}=1 / 2$ spins was the appropriate description[22]. It was further suggested that direct overlap of the $d$-orbitals generalizes this to a Heisenberg-Kitaev (HK) model[13], linearly interpolating between an isotropic Heisenberg model and Kitaev's bond-dependent exchange Hamiltonian. Extensive study of the HK model[23-28] has shown a variety of fascinating phenomena, including an extended spin liquid phase and quantum phase transitions into several well-understood magnetic ground states. While present, the zigzag phase seen in $\mathrm{Na}_{2} \mathrm{IrO}_{3}[2,4,6]$ is difficult to stabilize within the HK model; one must resort to additional $t_{2 g}-e_{g}$ exchange paths[18] or further neighbour hoppings[14]. In light of this puzzle one may question whether the HK model provides an adequate description of the honeycomb iridates even at the nearest neighbour level.

In this Letter, we show that when applied to the honeycomb iridates the $\mathrm{HK}$ model is incomplete, explicitly deriving the $j_{\text {eff }}=1 / 2$ spin model from a multiorbital $t_{2 g}$ HubbardKanamori Hamiltonian. Considering the most idealized crystal structure, an additional spin-spin interaction beyond the HK model must be included: bond-dependent symmetric offdiagonal exchange. The complete spin Hamiltonian has the form

$$
H=\sum_{\langle i j\rangle \in \alpha \beta(\gamma)}\left[J \vec{S}_{i} \cdot \vec{S}_{j}+K S_{i}^{\gamma} S_{j}^{\gamma}+\Gamma\left(S_{i}^{\alpha} S_{j}^{\beta}+S_{i}^{\beta} S_{j}^{\alpha}\right)\right],
$$

where $J$ is Heisenberg exchange, $K$ is the Kitaev exchange, and $\Gamma$ denotes the symmetric off-diagonal exchange. On each bond we distinguish one spin direction $\gamma$, labeling the bond

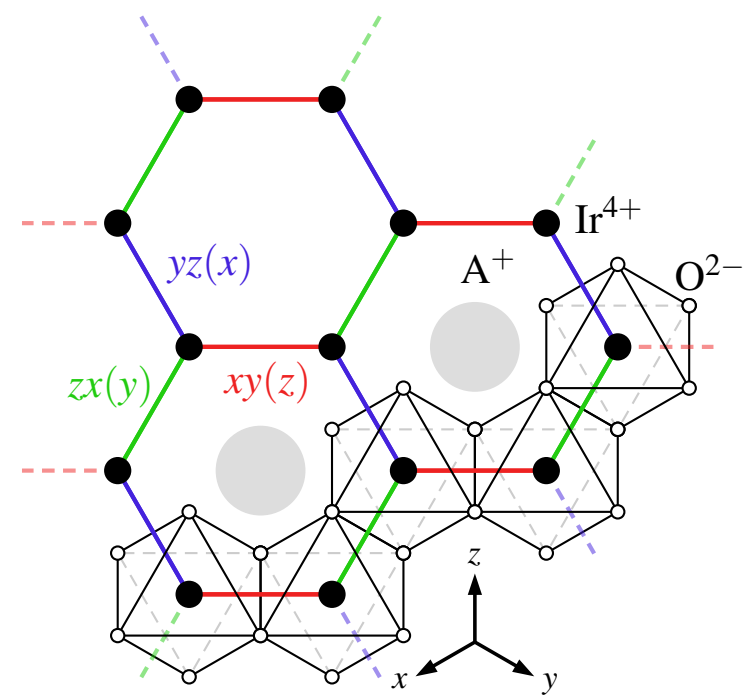

FIG. 1: Crystal structure of the honeycomb iridates $\mathrm{A}_{2} \mathrm{IrO}_{3}$ with $\mathrm{Ir}^{4+}$ in black, $\mathrm{O}^{2-}$ in white, and $\mathrm{A}=\mathrm{Na}^{+}, \mathrm{Li}^{+}$in gray. For the Kitaev and bond-dependent exchanges we have denoted the $y z(x)$ bonds blue, the $z x(y)$ bonds green and the $x y(z)$ bonds red.

$\alpha \beta(\gamma)$ where $\alpha$ and $\beta$ are the two remaining directions. Examining the phase diagram using a combination of classical arguments and exact diagonalization, we find that with the inclusion of $\Gamma$ new magnetic phases are stabilized near the Kitaev limits: an incommensurate spiral (IS) and $120^{\circ}$ order, in addition to extended regions of zigzag and stripy order.

Microscopics.- We first construct a minimal model of a honeycomb lattice of $\mathrm{Ir}^{4+}$ ions surrounded by a network of edge-sharing oxygen octahedra. The $\mathrm{Ir}^{4+} 5 d$ levels are split into an $e_{g}$ doublet and $t_{2 g}$ triplet by large crystal field effects, leaving a single hole in the $t_{2 g}$ states. Within the $t_{2 g}$ manifold, the orbital angular momentum behaves as an $l_{\text {eff }}=1$ triplet, with large spin-orbit coupling splitting this into an active $j_{\text {eff }}=1 / 2$ doublet and filled $j_{\text {eff }}=3 / 2$ states. Because of significant on-site interactions, localized $j_{\mathrm{eff}}=1 / 2$ spins provide an effective model for the low-energy physics. To per- 


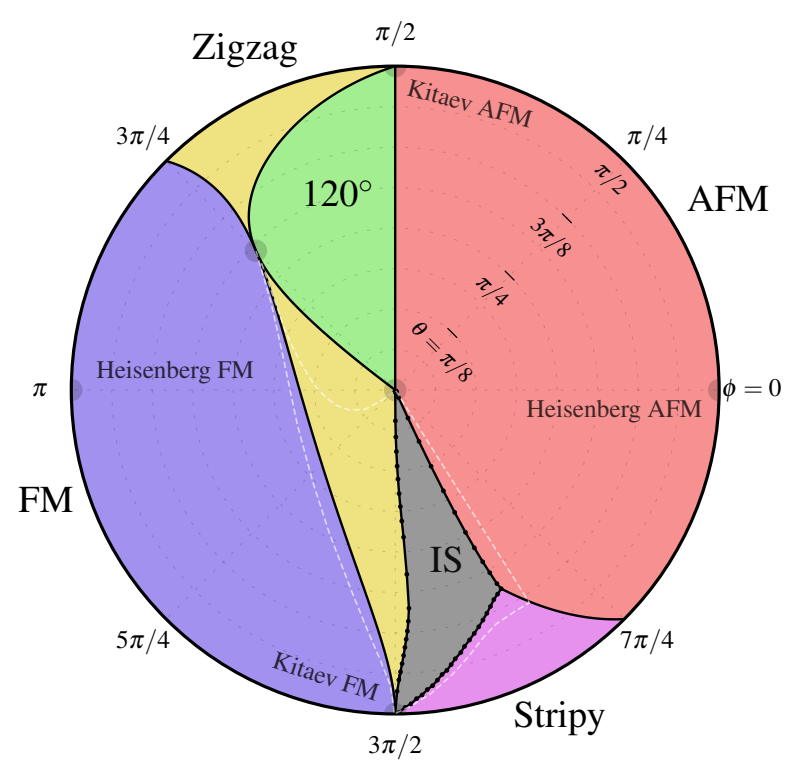

(a) Classical phase diagram with $\Gamma>0$

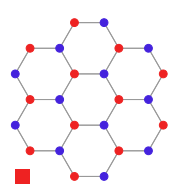

(b) AFM

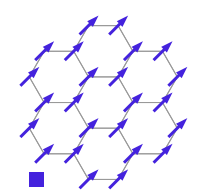

(c) FM

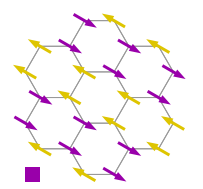

(d) Stripy

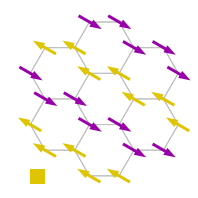

(e) Zigzag

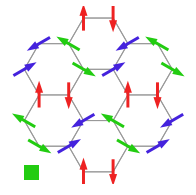

(f) $120^{\circ}$

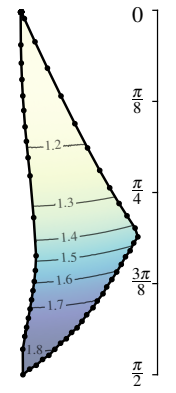

(g) $|\vec{Q}|$ in the IS
FIG. 2: (a) Combined Luttinger-Tisza and single- $Q$ analysis. Solid colours correspond to exact classical ground states from Luttinger-Tisza while the region indicated by the white dashed line are the single- $Q$ results. (b-f) Ground state spin configurations in each phase. $(\mathrm{g})$ Magnitude of the ordering wave-vector $\vec{Q}$ in the IS phase.

form the strong coupling expansion, we consider an atomic Hamiltonian of Kanamori form[29]:

$$
H_{0}=\sum_{i}\left[\frac{U-3 J_{H}}{2}\left(N_{i}-5\right)^{2}-2 J_{H} S_{i}^{2}-\frac{J_{H}}{2} L_{i}^{2}\right],
$$

where $N_{i}, S_{i}$, and $L_{i}$ are the total number, spin, and (effective) orbital angular momentum operators at site $i, U$ is the Coulomb interaction, and $J_{H}$ is Hund's coupling. The expansion is carried out in the limit $U, J_{H} \gg \lambda \gg t$, first taking $U$ and $J_{H}$ to be large. Since the spin-orbit coupling then dominates the kinetic terms, the resulting spin-orbital model can be projected into the $j_{\text {eff }}=1 / 2$ subspace.

The kinetic terms are encapsulated through a tight-binding model for the Ir $t_{2 g}$ orbitals, including both direct overlap of $d$ - orbitals and hopping mediated through the oxygen atoms. For our purposes, we focus on nearest-neighbour bonds where we then have

$$
\sum_{\langle i j\rangle \in \alpha \beta(\gamma)}\left[t_{1}\left(d_{i \alpha}^{\dagger} d_{j \alpha}+d_{i \beta}^{\dagger} d_{j \beta}\right)+t_{2}\left(d_{i \alpha}^{\dagger} d_{j \beta}+d_{i \beta}^{\dagger} d_{j \alpha}\right)+t_{3} d_{i \gamma}^{\dagger} d_{i \gamma}\right],
$$

where $d_{i \alpha}^{\dagger}=\left(d_{i \alpha \uparrow}^{\dagger} d_{i \alpha \downarrow}^{\dagger}\right)$ and $d_{i \alpha}$ are the creation and annihilation operators for the $t_{2 g}$ state $\alpha$ at site $i$. Here we sum over the $y z(x), z x(y)$ and $x y(z)$ links as indicated in Fig. 1, but mapping the directions to orbitals as $x \rightarrow y z, y \rightarrow z x$ and $z \rightarrow x y$. The parameters $t_{1}, t_{2}$, and $t_{3}$ are given by

$$
t_{1}=\frac{t_{d d \pi}+t_{d d \delta}}{2}, t_{2}=\frac{t_{p d \pi}^{2}}{\Delta_{p d}}+\frac{t_{d d \pi}-t_{d d \delta}}{2}, t_{3}=\frac{3 t_{d d \sigma}+t_{d d \delta}}{4},
$$

where $t_{d d \sigma}, t_{d d \pi}, t_{d d \delta}$ and $t_{p d \pi}$ are Slater-Koster[30] parameters for the direct Ir-Ir overlap and Ir-O overlap while $\Delta_{p d}$ is the Ir$\mathrm{O}$ gap [31]. Treating the kinetic terms as a perturbation yields the Hamiltonian in Eq. 1 with

$$
\begin{aligned}
J & =\frac{4}{27}\left[\frac{6 t_{1}\left(t_{1}+2 t_{3}\right)}{U-3 J_{H}}+\frac{2\left(t_{1}-t_{3}\right)^{2}}{U-J_{H}}+\frac{\left(2 t_{1}+t_{3}\right)^{2}}{U+2 J_{H}}\right], \\
K & =\frac{8 J_{H}}{9}\left[\frac{\left(t_{1}-t_{3}\right)^{2}-3 t_{2}^{2}}{\left(U-3 J_{H}\right)\left(U-J_{H}\right)}\right], \\
\Gamma & =\frac{16 J_{H}}{9}\left[\frac{t_{2}\left(t_{1}-t_{3}\right)}{\left(U-3 J_{H}\right)\left(U-J_{H}\right)}\right] .
\end{aligned}
$$

Exchanges of the same form as the $\Gamma$ term were originally called symmetric anisotropic exchange[32,33] and can be related to the truncated dipolar exchange[34, 35] discussed in other contexts through a reparametrization. We stress that since this term is allowed by symmetry even in the most idealized cases, the presence of the $\Gamma$ term is a generic feature of $j_{\text {eff }}=1 / 2$ models with edge-shared octahedra (see the Supplemental material [36] for more information). To confirm this, the strong coupling expansion was also carried out in the limit where $U, \lambda \gg J_{H} \gg t$, with the contributions of $J_{H}$ included in the excited states perturbatively. While energies of the virtual states involve $\lambda$ instead of $J_{H}$, all three terms are generated, with the dependence of $K$ and $\Gamma$ on the hoppings $t_{1}$, $t_{2}$, and $t_{3}$ unchanged (Supplemental Material [36]). Whereas the Kitaev limit can be naturally accessed when $t_{2} \gg t_{1}, t_{3}$, leaving this regime introduces both $J$ and $\Gamma$ making it difficult to reach the HK limit[37]. Fine tuning could in principle render $\Gamma$ small, but the dominant contributions to $t_{1} \sim t_{d d \pi}$ and $t_{3} \sim t_{d d \sigma}$ are of opposite sign making any such tuning implausible. Further applications to wider classes of iridium oxides are left for future work.

Classical phase diagram.- To understand the effects of including this bond-dependent $\Gamma$ term, we first map out the classical magnetic phases. We parametrize the exchanges using angles $\phi$ and $\theta$

$$
J=\sin \theta \cos \phi, \quad K=\sin \theta \sin \phi, \quad \Gamma=\cos \theta,
$$

fixing the energy scale so that $\sqrt{J^{2}+K^{2}+\Gamma^{2}}=1$. By mapping $\vec{S}_{i} \rightarrow-\vec{S}_{i}$ on one sublattice, we send $\phi \rightarrow-\phi$ and 
$\theta \rightarrow \pi-\theta$, so we can consider only $\Gamma>0$. To obtain the classical phase diagram, the Luttinger-Tisza approximation[38, 39] is first used. In this approximation, the constraint of fixed spin length is released, allowing for a direct solution of the classical model. In the regions of the phase diagram where this fails, we have further supplemented this with an analysis of a single- $Q$ ansatz. The combined results are shown in Fig. 2a with $0<\theta \leq \pi / 2$ mapped to the radial direction and $0 \leq \phi<2 \pi$ mapped to the angular direction.

When the resulting spin configuration satisfies the local length constraint, the Luttinger-Tisza method yields the exact classical ground state. This holds for most of the phase diagram aside from the region in Fig. 2a indicated by dashed white lines. In this region we consider spin configurations of the form

$$
\vec{S}_{i}=\sin \eta_{i}\left[\hat{e}_{i}^{x} \cos \left(\vec{Q} \cdot \vec{r}_{i}\right)+\hat{e}_{i}^{y} \sin \left(\vec{Q} \cdot \vec{r}_{i}\right)\right]+\cos \eta_{i} \hat{e}_{i}^{z}
$$

where the canting angles $\eta_{i}$ and local frames defined by $\left(\hat{e}_{i}^{x}, \hat{e}_{i}^{y}, \hat{e}_{i}^{z}\right)$ are independent variational parameters on two sublattices. The energy of the ansatz is minimized over the variational parameters and $\vec{Q}$ for each pair of angles $(\phi, \theta)$.

In the HK limit [the boundary of the disk in Fig 2a] there are four classical phases: the ferromagnet (FM), antiferromagnet (AFM), stripy, and zigzag as in Figs. 2b-2e. These states occupy large regions of phase space even as $\Gamma$ is introduced, with the AFM and FM states covering most the phase diagram. Finite $\Gamma$ breaks the accidental spin rotational symmetry enjoyed by the FM and AFM states in the (classical) HK limit, pinning the orderings to fixed spatial direction. For $\Gamma>0$, the AFM becomes pinned along the [111] direction whereas the FM lies in the plane perpendicular to [111] with all directions degenerate. The stripy and zigzag phases have the spins in direction $x, y$ or $z$ locked to the orientations of the stripe and zigzag pattern, tilting slightly away from the stripe and zigzag direction as $\Gamma$ becomes non zero.

The effects of $\Gamma$ are most evident where a large classical degeneracy is present, such as near the Kitaev points at $(\phi, \theta)=( \pm \pi / 2, \pi / 2)$ and near $(0,0)$, where we only have the bond-dependent $\Gamma$ term. Here two new states are introduced: $120^{\circ}$ order and an incommensurate spiral. The $120^{\circ}$ order with wave vector $\vec{Q}=K$ appears near the (antiferromagnetic) Kitaev limit at $(\pi / 2, \pi / 2)$. This is a coplanar spiral, with the spins lying in the plane perpendicular to [111]. The spins are at relative angles $0, \pm 2 \pi / 3$ on the same sublattice (as shown in Fig. 2f), with the relative angle between sublattices unconstrained. An additional degenerate point appears at $(\phi, \theta)=\left(3 \pi / 4, \cos ^{-1}\left(\frac{1}{\sqrt{3}}\right)\right)$ where $J=-K=-\Gamma$, with the $120^{\circ}$, FM, and zigzag phases meeting at a single point[40]. The second large region of zigzag phase appearing when $\Gamma \gg|J|,|K|$ has the spins predominantly oriented along the [1 $1 \overline{1}]$, [ $[\overline{1} 1 \overline{1}]$, and [ $[\overline{1} 11]$ directions, tilting away slightly as one explores the phase. The IS phase remains coplanar despite the $\vec{Q}$ vector varying throughout the phase. The magnitude of the IS wave vector lies in the range $1.2<|\vec{Q}|<1.8$ as shown in Fig. 2g.

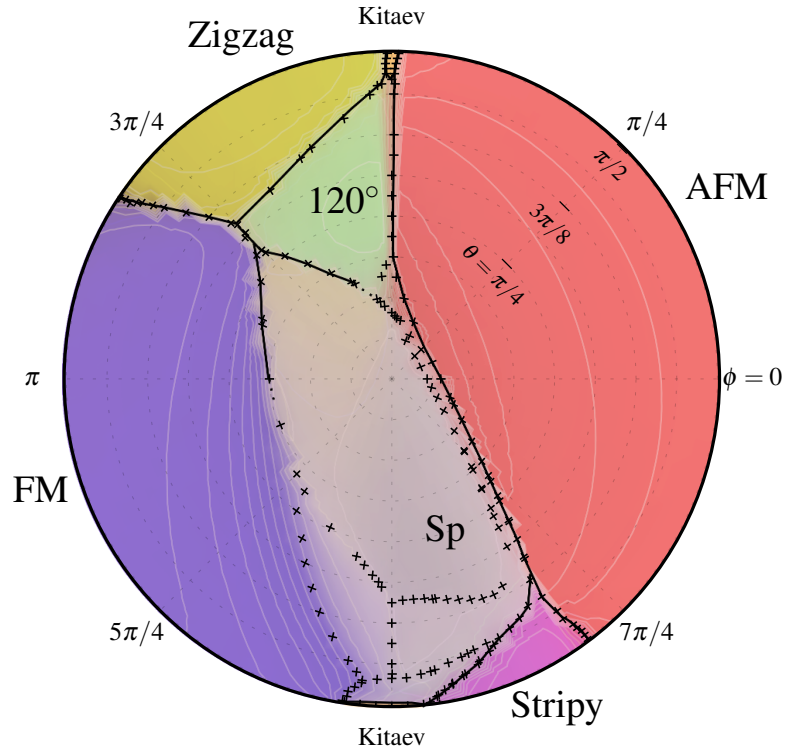

(a) Phase diagram for $\Gamma>0$

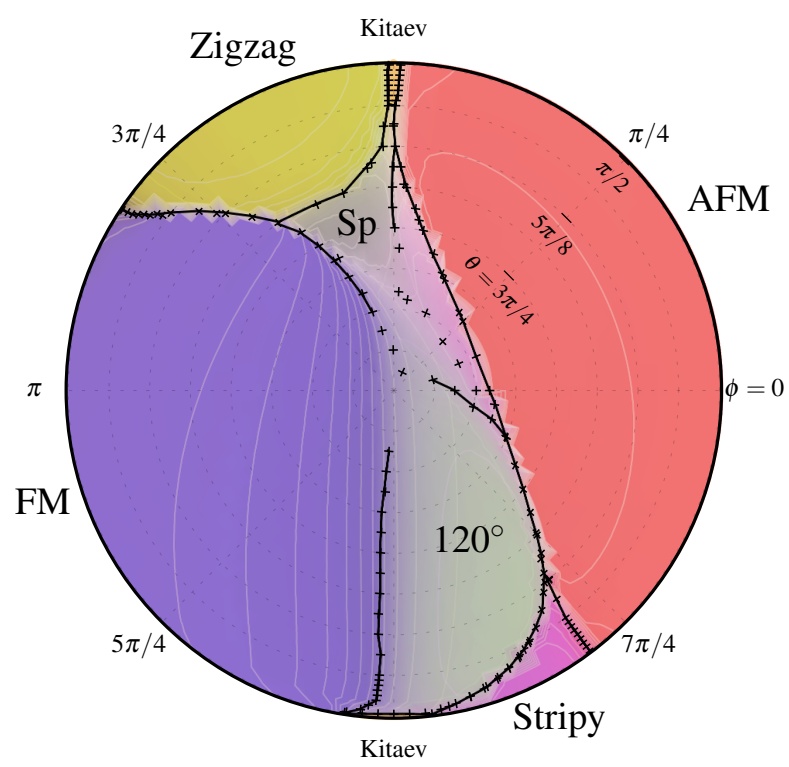

(b) Phase diagram for $\Gamma<0$

FIG. 3: [(a),(b)] Phase boundaries from exact diagonalization of a 24-site cluster. Markers indicate the location of singular features in $-\partial^{2} E / \partial \phi^{2}$ or $-\partial^{2} E / \partial \theta^{2}$, with lines to guide the eye along the sharp phase boundaries. Colours [as in Fig. 2a] and contours map magnitudes of the static structure factor. [as in Eq. 8] for each phase. The Kitaev spin liquid is shown in orange, whereas the spiral phase is shown in dark gray. The HK limit lies at the boundary of each disk at $\theta=\pi / 2$.

Exact diagonalization.- To gain an understanding of the features of the classical results that carry over to the full quantum mechanical model we have performed exact diagonaliza- 
tion. We consider a 24-site cluster that has been used previously to study the $\mathrm{HK}$ model[13, 18, 19], providing a reasonable description of the phases found at the classical level as well as the Kitaev spin liquids. In the HK limit, the existence of a local spin rotation $[13,41]$ that maps $J \rightarrow-J$ and $K \rightarrow K+2 J$, gives four well-understood magnetic limits in addition to the two exactly solvable Kitaev points. These are the FM, AFM, and their zigzag and stripy images under the mapping. This transformation is no longer useful as $\Gamma$ is included[42], but the phases surrounding these points can still be identified with each respective limit. While the IS phase is unlikely to be well represented on such a small cluster, the remaining phases such as the $120^{\circ}$ phase are compatible with the cluster geometry. We note that the transformation used to relate $\Gamma>0$ to $\Gamma<0$ no longer applies in the quantum case and so both regions must be analyzed separately.

To identify the phase boundaries, we have computed the second derivatives of the ground-state energy, $-\partial^{2} E / \partial \phi^{2}$ and $-\partial^{2} E / \partial \theta^{2}$, looking for singular features that indicate changes in the ground state characteristics. Phases containing exactly solvable or well-understood points, such as the zigzag, stripy, AFM, FM, and the Kitaev spin liquids can be readily identified. The remaining phases were identified by examining the spin-spin correlation functions $\left\langle S_{i}^{\alpha} S_{j}^{\beta}\right\rangle$, primarily through the static structure factor

$$
S_{Q}=\frac{1}{N} \sum_{i j} e^{i \vec{Q} \cdot\left(\vec{r}_{i}-\vec{r}_{j}\right)}\left\langle\vec{S}_{i} \cdot \vec{S}_{j}\right\rangle
$$

in both the original basis and after applying the local spin rotation discussed above[13]. The resulting phase diagrams for $\Gamma>0$ and $\Gamma<0$ are presented in Fig. 3, with the structure factor for each phase plotted using the colours from Fig. 2a and then overlayed. Contours indicating lines of constant $S_{Q}$ in each phase are also shown. The phase diagrams bear a remarkable resemblance to the classical results, with the gross features of the phase diagram preserved for both $\Gamma>0$ and $\Gamma<0$.

While the new $120^{\circ}$ phase was identified, the results are less distinctive in the regions where the Luttinger-Tisza approach failed. Because of the suggestion of incommensurate phases from the classical analysis, it is likely that the small size of cluster used may not properly capture the behaviour in this region. Nevertheless, in the classical IS region exact diagonalization shows a spiral phase $(\mathrm{Sp})$ with correlations at wave vector $\vec{Q}=K / 2$, the ordering with the longest periodicity allowed by the cluster size. This wave vector has magnitude $|\vec{Q}| \approx 1.2$, roughly in line with the range prescribed by the classical calculations. The neighbouring regions are also well defined, with stripy correlations for $\Gamma<0$ and zigzag correlations for $\Gamma>0$, as expected from the classical analysis. While the stripy correlations for $\Gamma<0$ are quite strong, the corresponding zigzag correlations for $\Gamma>0$ are weak, showing no sharp transition as one moves into the classical IS region. At the pure $\Gamma$ limits the correlators become short ranged, with most of the correlators exactly zero. From these results we expect the gross features of the phase diagram to be robust to finite size effects except perhaps for the wave vector of the spiral phase.

Discussion.- Within the scope of the model presented, the zigzag phase observed in $\mathrm{Na}_{2} \mathrm{IrO}_{3}[2,4,6]$ appears only when $J$ is negative. This is plausible: in Eq. 3 take the Slater-Koster parameters to have the canonical ratios $t_{d d \sigma}: t_{d d \pi}=3:-2$ (assuming that $\left.t_{d d \delta} \sim 0\right)$ then $2 t_{1}+t_{3} \sim 0$ and $t_{1}\left(t_{1}+2 t_{3}\right)<0$ giving $J<0$ at leading order in $J_{H} / U$. Additional contributions to these exchanges, such as on-site oxygen interactions[13] and $t_{2 g}-e_{g}$ contributions[18] possibly affect the details. Further, $a b$ initio calculations of the electronic band structure of $\mathrm{Na}_{2} \mathrm{IrO}_{3}[16,17,20]$ and $\mathrm{Li}_{2} \mathrm{IrO}_{3}[43]$ suggest that second and third neighbour hoppings as well as trigonal and other structural distortions may not be negligible. Some consequences of further neighbour exchange have been discussed[14], but a proper treatment is missing - one must include the orbital dependence of these hoppings that results in anisotropic exchanges. Inclusion of trigonal and other distortions allows an additional symmetric off-diagonal exchange[44], but these have been estimated to be small experimentally[11]. We further expect that the nearest neighbour model dominates over the longer range exchanges, and so including them should not alter the results qualitatively.

We emphasize that understanding the minimal model introduced in this work is the first step towards a complete picture of the honeycomb iridates. Evidence of symmetric offdiagonal exchange can be seen through anisotropy in the magnetic susceptibility. From a high-temperature expansion of the model in Eq. 1, one finds $\left(\Theta_{\perp}-\Theta_{\|}\right) /\left(\Theta_{\perp}+2 \Theta_{\|}\right)=\Gamma /(3 J+K)$ independent of $g$-factor anisotropy, where $\Theta_{\|}$and $\Theta_{\perp}$ are the Curie-Weiss temperatures for the in- and out-of-plane susceptibilities. Fitting to experimental data for $\mathrm{Na}_{2} \mathrm{IrO}_{3}$ [1] yields the relation $\Gamma /(3 J+K) \sim-0.3$, showing that if we are near the zigzag regime where $K \gg|J|$ then there is non-negligible $\Gamma$ exchange. Given that $120^{\circ}$ and IS order appear in proximity to the zigzag phase, these could be promising candidates for ordering in other honeycomb iridates such as $\mathrm{Li}_{2} \mathrm{IrO}_{3}$.

Note added.- After submission of this work, the existence of the $\Gamma$ term was discussed based on $a b$ initio quantum chemistry calculations in Ref. [45]

Acknowledgements.- We thank R. Schaffer, K. Hwang, V. Vijay Shankar and Y. B. Kim for useful discussions. Computations were performed on the GPC supercomputer at the SciNet HPC Consortium. SciNet is funded by: the Canada Foundation for Innovation under the auspices of Compute Canada, the Government of Ontario, Ontario Research Fund - Research Excellence; and the University of Toronto. This work was supported by the NSERC of Canada, CIFAR, and the Centre for Quantum Materials at the University of Toronto.

* Electronic Address: hykee@physics.utoronto.ca

[1] Y. Singh and P. Gegenwart, Phys. Rev. B 82, 064412 (2010). 
[2] X. Liu, T. Berlijn, W.-G. Yin, W. Ku, A. Tsvelik, Y.-J. Kim, H. Gretarsson, Y. Singh, P. Gegenwart, and J. Hill, Phys. Rev. B 83, 220403 (2011).

[3] Y. Singh, S. Manni, J. Reuther, T. Berlijn, R. Thomale, W. Ku, S. Trebst, and P. Gegenwart, Phys. Rev. Lett. 108, 127203 (2012).

[4] F. Ye, S. Chi, H. Cao, B. C. Chakoumakos, J. A. FernandezBaca, R. Custelcean, T. Qi, O. Korneta, and G. Cao, Phys. Rev. B 85, 180403 (2012).

[5] S. Lovesey and A. Dobrynin, J. Phys. Condens. Matter 24, 382201 (2012).

[6] S. K. Choi, R. Coldea, A. N. Kolmogorov, T. Lancaster, I. I. Mazin, S. J. Blundell, P. G. Radaelli, Y. Singh, P. Gegenwart, K. R. Choi, S.-W. Cheong, P. J. Baker, C. Stock, and J. Taylor, Phys. Rev. Lett. 108, 127204 (2012).

[7] R. Comin, G. Levy, B. Ludbrook, Z.-H. Zhu, C. N. Veenstra, J. A. Rosen, Y. Singh, P. Gegenwart, D. Stricker, J. N. Hancock, D. van der Marel, I. S. Elfimov, and A. Damascelli, Phys. Rev. Lett. 109, 266406 (2012).

[8] J. P. Clancy, N. Chen, C. Y. Kim, W. F. Chen, K. W. Plumb, B. C. Jeon, T. W. Noh, and Y.-J. Kim, Phys. Rev. B 86, 195131 (2012).

[9] H. Gretarsson, J. P. Clancy, Y. Singh, P. Gegenwart, J. P. Hill, J. Kim, M. H. Upton, A. H. Said, D. Casa, T. Gog, and Y.-J. Kim, Phys. Rev. B 87, 220407 (2013).

[10] H. Gretarsson, J. P. Clancy, X. Liu, J. P. Hill, E. Bozin, Y. Singh, S. Manni, P. Gegenwart, J. Kim, A. H. Said, D. Casa, T. Gog, M. H. Upton, H.-S. Kim, J. Yu, V. M. Katukuri, L. Hozoi, J. van den Brink, and Y.-J. Kim, Phys. Rev. Lett. 110, 076402 (2013).

[11] G. Cao, T. Qi, L. Li, J. Terzic, V. Cao, S. Yuan, M. Tovar, G. Murthy, and R. Kaul, Physical Review B 88, 220414 (2013).

[12] A. Shitade, H. Katsura, J. Kuneš, X.-L. Qi, S.-C. Zhang, and N. Nagaosa, Phys. Rev. Lett. 102, 256403 (2009).

[13] J. Chaloupka, G. Jackeli, and G. Khaliullin, Phys. Rev. Lett. 105, 027204 (2010).

[14] I. Kimchi and Y.-Z. You, Phys. Rev. B 84, 180407 (2011).

[15] S. Bhattacharjee, S.-S. Lee, and Y. B. Kim, New J. Phys. 14, 073015 (2012).

[16] I. Mazin, H. O. Jeschke, K. Foyevtsova, R. Valentí, and D. Khomskii, Phys. Rev. Lett. 109, 197201 (2012).

[17] C. H. Kim, H. S. Kim, H. Jeong, H. Jin, and J. Yu, Phys. Rev. Lett. 108, 106401 (2012).

[18] J. Chaloupka, G. Jackeli, and G. Khaliullin, Phys. Rev. Lett. 110, 097204 (2013).

[19] S. Okamoto, Phys. Rev. B 87, 064508 (2013).

[20] K. Foyevtsova, H. O. Jeschke, I. I. Mazin, D. I. Khomskii, and R. Valentí, Phys. Rev. B 88, 035107 (2013).

[21] A. Kitaev, Ann. Phys. 321, 2 (2006).

[22] G. Jackeli and G. Khaliullin, Phys. Rev. Lett. 102, 017205 (2009).

[23] H.-C. Jiang, Z.-C. Gu, X.-L. Qi, and S. Trebst, Phys. Rev. B 83, 245104 (2011).

[24] J. Reuther, R. Thomale, and S. Trebst, Phys. Rev. B 84, 100406 (2011).

[25] F. Trousselet, G. Khaliullin, and P. Horsch, Phys. Rev. B 84, 054409 (2011).

[26] R. Schaffer, S. Bhattacharjee, and Y. B. Kim, Phys. Rev. B 86, 224417 (2012).

[27] C. Price and N. B. Perkins, Phys. Rev. Lett. 109, 187201 (2012).

[28] C. Price and N. B. Perkins, Phys. Rev. B 88, 024410 (2013).

[29] S. Sugano, Multiplets of transition-metal ions in crystals (Academic Press, 1970).

[30] J. C. Slater and G. F. Koster, Phys. Rev. 94, 1498 (1954).
[31] An additional $x y-x z$ and $x y-y z$ hopping is allowed by the full crystal symmetry, but is not present when we consider loworder processes involving only two neighbouring Ir atoms with ideal oxygen octahedra.

[32] T. Moriya, Phys. Rev. 120, 91 (1960).

[33] I. Dzyaloshinsky, J. Phys. Chem. Solids 4, 241 (1958).

[34] J. S. Gardner, M. J. P. Gingras, and J. E. Greedan, Rev. Mod. Phys. 82, 53 (2010).

[35] A. van Rynbach, S. Todo, and S. Trebst, Phys. Rev. Lett. 105, 146402 (2010).

[36] See the Supplementary material attached for a discussion of the strong coupling limits presented in the main text and an explicit derivation of the $\Gamma$ term.

[37] Taking $t_{2}=0$ and $t_{1} \neq t_{3}$ gives the HK model, but this corresponds to the case of corner-shared octahedra, as discussed in [46]. Reaching this point in the edge-shared case again requires unrealistic fine-tuning.

[38] J. Luttinger and L. Tisza, Phys. Rev. 70, 954 (1946).

[39] D. Litvin, Physica 77, 205 (1974).

[40] At this point the spin Hamiltonian takes a truncated dipolar form $\sim \sum_{\langle i j\rangle}\left(\vec{\delta}_{i j} \cdot \vec{S}_{i}\right)\left(\vec{\delta}_{i j} \cdot \vec{S}_{j}\right)$ where $\vec{\delta}_{i j}$ is the bond direction vector.

[41] G. Khaliullin, Prog. Theor. Phys. Suppl. 160, 155 (2005).

[42] If one performs the site dependent rotation with the $\Gamma$ term included the resulting Hamiltonian is not of the same form, with the $\Gamma$ parts taking on a sign structure with an enlarged unit cell.

[43] H.-S. Kim, C. H. Kim, H. Jeong, H. Jin, and J. Yu, Phys. Rev. B 87, 165117 (2013).

[44] The additional symmetric off-diagonal exchange has the form $\Gamma^{\prime} \sum_{\langle i j\rangle \in \alpha \beta(\gamma)}\left(S_{i}^{\alpha} S_{j}^{\gamma}+S_{i}^{\gamma} S_{j}^{\alpha}+S_{i}^{\beta} S_{j}^{\gamma}+S_{i}^{\gamma} S_{j}^{\beta}\right)$.

[45] V. M. Katukuri, S. Nishimoto, V. Yushankhai, A. Stoyanova, H. Kandpal, S. Choi, R. Coldea, I. Rousochatzakis, L. Hozoi, and J. v. d. Brink, arXiv:1312.7437 (2013).

[46] S. Okamoto, Phys. Rev. Lett. 110, 066403 (2013). 


\title{
Supplementary material for "Generic Spin Model for the Honeycomb Iridates beyond the Kitaev Limit"
}

\author{
Jeffrey G. Rau, ${ }^{1}$ Eric Kin-Ho Lee, ${ }^{1}$ and Hae-Young Kee ${ }^{1,2, *}$ \\ ${ }^{1}$ Department of Physics, University of Toronto, Toronto, Ontario M5S 1A7, Canada \\ ${ }^{2}$ Canadian Institute for Advanced Research/Quantum \\ Materials Program, Toronto, Ontario MSG 1Z8, Canada
}




\section{TIGHT-BINDING MODEL FOR EDGE-SHARING OCTAHEDRA}

Consider a single $x y(z)$ type bond, placing the $\operatorname{Ir}^{4+}$ ions at the origin and along $\hat{y}-\hat{x}$ as shown in Fig. 1. The remaining bonds can generated using lattice symmetries. We denote the corresponding $t_{2 g}$ electron operators as $d_{1}^{\dagger}=\left(d_{1, y z}^{\dagger} d_{1, z x}^{\dagger} d_{1, x y}^{\dagger}\right)$ and $d_{2}^{\dagger}=\left(d_{2, y z}^{\dagger} d_{2, z x}^{\dagger} d_{2, x y}^{\dagger}\right)$. The bond Hamiltonian can be seen to be

$$
T+T^{\dagger}=d_{1}^{\dagger} T_{12} d_{2}+d_{2}^{\dagger} T_{21} d_{1}
$$

where $T_{21}=T_{12}^{\dagger}$. Due to inversion about the bond center and time-reversal, in the $t_{2 g}$ basis we have that $T_{12}=T_{12}^{\dagger}=T_{12}^{*}$ so $T_{12}$ is real and symmetric.

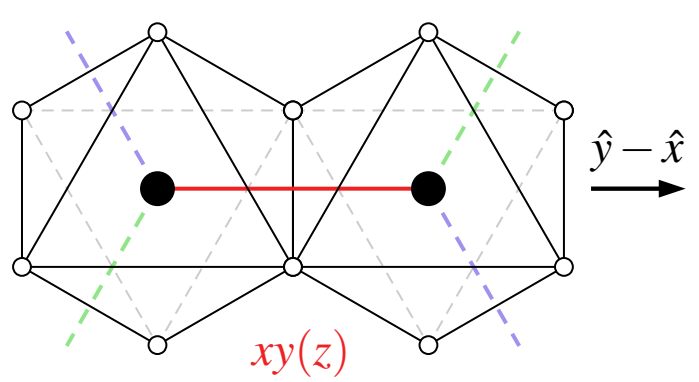

FIG. 1: The $x y(z)$ bond on which we will compute the effective Hamiltonian

When only the two $\mathrm{Ir}^{4+}$ ions and the octahedra of $\mathrm{O}^{2-}$ ions are included the form of $T_{12}$ is constrained by symmetry. These symmetries are inversion through the bond center, as well as $C_{2}$ axes through the [110], [110] and [001] axes, giving the form

$$
T_{12}=\left(\begin{array}{lll}
t_{1} & t_{2} & 0 \\
t_{2} & t_{1} & 0 \\
0 & 0 & t_{3}
\end{array}\right)
$$

with the three independent real parameters $t_{1}, t_{2}$ and $t_{3}$. The actual bond symmetry in the crystal can be lower, for example due to trigonal distortion. Within the $t_{2 g}$ manifold this can introduce additional $x z-x y$ and $y z-x y$ hoppings such as

$$
T_{12}=\left(\begin{array}{lll}
t_{1} & t_{2} & t_{4} \\
t_{2} & t_{1} & t_{4} \\
t_{4} & t_{4} & t_{3}
\end{array}\right)
$$

\footnotetext{
* Electronic Address: hykee@physics.utoronto.ca
} 


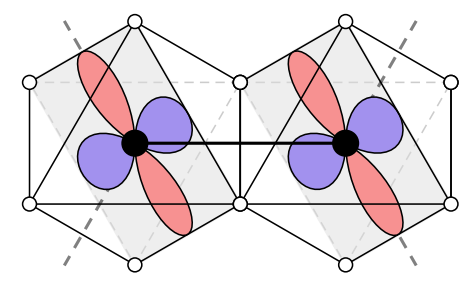

(a) Ir-Ir overlap for $t_{1}$

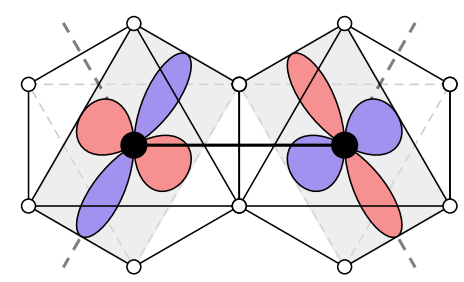

(c) Ir-Ir overlap for $t_{2}$

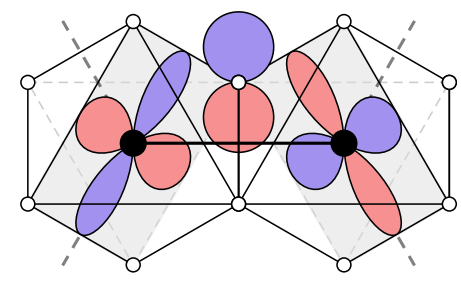

(b) Ir-O-Ir overlap for $t_{2}$

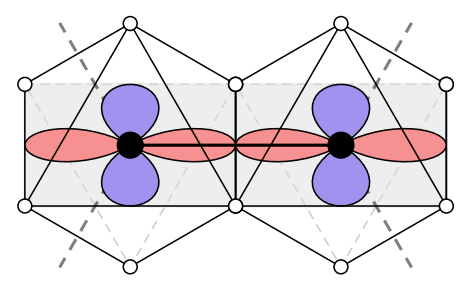

(d) Ir-Ir overlap for $t_{3}$

FIG. 2: Schematic visual representation of the types orbital overlap contributing to the hoppings $t_{1}, t_{2}$ and $t_{3}$ in Eq. 4 a for the $x y(z)$ bond.

We will discuss two possibilities relevant for $\mathrm{Na}_{2} \mathrm{IrO}_{3}$ and $\mathrm{Li}_{2} \mathrm{IrO}_{3}$ : direct overlap between the $d$ orbitals and hopping mediated through the $\mathrm{O}^{2-}$ ions.

$$
\begin{aligned}
& t_{1}=\frac{1}{2}\left(t_{d d \pi}+t_{d d \delta}\right) \\
& t_{2}=\frac{1}{2}\left(t_{d d \pi}-t_{d d \delta}\right)+\frac{t_{p d \pi}^{2}}{\Delta_{p d}} \\
& t_{3}=\frac{1}{4}\left(3 t_{d d \sigma}+t_{d d \delta}\right)
\end{aligned}
$$

The three parameters $t_{d d \sigma}, t_{d d \pi}$ and $t_{d d \delta}$ are the usual Slater-Koster parameters for direct $d$-orbital overlap, with one expecting $\left|t_{d d \sigma}\right|>\left|t_{d d \pi}\right|>\left|t_{d d \delta}\right|$. The oxygen mediated hopping is through the $t_{p d \pi}$ overlap with $\Delta_{p d}$ being the chemical potential difference between the $\mathrm{Ir}^{4+}$ and $\mathrm{O}^{2-}$ ions. A visual representation of these overlaps is shown in Fig. 2.

\section{STRONG COUPLING EXPANSIONS}

To derive the effective Hamiltonian for the $j_{\mathrm{eff}}=1 / 2$ states we consider two forms of strong coupling expansion. The first is the conventional case, where we take $U, J_{H} \gg \lambda \gg t$. We consider the Kanamori Hamiltonian for the two atoms

$$
H_{1}+H_{2}=\frac{U-3 J_{H}}{2}\left[\left(N_{1}-5\right)^{2}+\left(N_{2}-5\right)^{2}\right]-2 J_{H}\left(S_{1}^{2}+S_{2}^{2}\right)-\frac{J_{H}}{2}\left(L_{1}^{2}+L_{2}^{2}\right)
$$


where $U$ is the (screened) Coulomb interaction, $J_{H}$ is Hund's coupling, $N$ is the total density operator, $L$ is the total (effective) angular momentum operator and $S$ is the total spin operator.

Treating $T+T^{\dagger}$ as a perturbation to $H_{1}+H_{2}$, an effective Hamiltonian within the $j_{\text {eff }}=1 / 2$ subspace can be determined

$$
H_{\mathrm{eff}} \sim-\sum_{\alpha \beta} \sum_{n \neq 0}\left[\frac{\left\langle\alpha\left|T^{\dagger}\right| n\right\rangle\langle n|T| \beta\rangle}{E_{n}-E_{0}}+\frac{\langle\alpha|T| n\rangle\left\langle n\left|T^{\dagger}\right| \beta\right\rangle}{E_{n}-E_{0}}\right]|\alpha\rangle\langle\beta|
$$

where $\alpha, \beta$ run over $j_{\text {eff }}=1 / 2$ states, $\left|+\frac{1}{2},+\frac{1}{2}\right\rangle,\left|+\frac{1}{2}-\frac{1}{2}\right\rangle,\left|-\frac{1}{2}+\frac{1}{2}\right\rangle$ and $\left|-\frac{1}{2},-\frac{1}{2}\right\rangle$. The excited states of $H_{1}+H_{2}$ are denoted by $|n\rangle$ with energies $E_{n}$ greater than the ground state energy $E_{0}$. By symmetry, the resulting spin Hamiltonian takes the form

$$
H_{\mathrm{eff}} \sim J \vec{S}_{1} \cdot \vec{S}_{2}+K S_{1}^{z} S_{2}^{z}+\Gamma\left(S_{1}^{x} S_{2}^{y}+S_{1}^{y} S_{2}^{x}\right)+\Gamma^{\prime}\left(S_{1}^{x} S_{2}^{z}+S_{1}^{z} S_{2}^{x}+S_{1}^{y} S_{2}^{z}+S_{1}^{z} S_{2}^{y}\right)
$$

Carrying out the expansion one arrives at the following expressions for the exchange constants

$$
\begin{aligned}
J & =\frac{4}{27}\left[\frac{6 t_{1}\left(t_{1}+2 t_{3}\right)-9 t_{4}^{2}}{U-3 J_{H}}+\frac{9 t_{4}^{2}+2\left(t_{1}-t_{3}\right)^{2}}{U-J_{H}}+\frac{\left(2 t_{1}+t_{3}\right)^{2}}{U+2 J_{H}}\right] \\
& \approx \frac{4}{9 U}\left[\left(2 t_{1}+t_{3}\right)^{2}+\frac{2 J_{H}}{U}\left[2 t_{1}\left(t_{1}+2 t_{3}\right)-3 t_{4}^{2}\right]\right]+O\left(\frac{J_{H}^{2}}{U^{2}}\right) \\
K & =\frac{8 J_{H}}{9}\left[\frac{\left(t_{1}-t_{3}\right)^{2}-3\left(t_{2}^{2}-t_{4}^{2}\right)}{\left(U-3 J_{H}\right)\left(U-J_{H}\right)}\right] \approx \frac{8}{9 U}\left(\frac{J_{H}}{U}\right)\left[\left(t_{1}-t_{3}\right)^{2}-3\left(t_{2}^{2}-t_{4}^{2}\right)\right]+O\left(\frac{J_{H}^{2}}{U^{2}}\right) \\
\Gamma & =\frac{8 J_{H}}{9}\left[\frac{3 t_{4}^{2}+2 t_{2}\left(t_{1}-t_{3}\right)}{\left(U-3 J_{H}\right)\left(U-J_{H}\right)}\right] \approx \frac{8}{9 U}\left(\frac{J_{H}}{U}\right)\left[3 t_{4}^{2}+2 t_{2}\left(t_{1}-t_{3}\right)\right]+O\left(\frac{J_{H}^{2}}{U^{2}}\right) \\
\Gamma^{\prime} & =-\frac{8 J_{H}}{9}\left[\frac{t_{4}\left(t_{1}-t_{3}-3 t_{2}\right)}{\left(U-3 J_{H}\right)\left(U-J_{H}\right)}\right] \approx-\frac{8}{9 U}\left(\frac{J_{H}}{U}\right)\left[t_{4}\left(t_{1}-t_{3}-3 t_{2}\right)\right]+O\left(\frac{J_{H}^{2}}{U^{2}}\right)
\end{aligned}
$$

The full nearest neighbour spin model can be obtained using lattice symmetries to generate the remaining bonds. The expressions given in Eqs. 3-5 of the main text can be obtained from Eq. 8 by setting $t_{4}=0$.

A similar calculation can be carried out taking the limit $U, \lambda \gg J_{H} \gg t$. Here the atomic Hamiltonian includes only the Coulomb interaction $U$ and spin-orbit coupling $\lambda$. The contributions proportional to $J_{H}$ can then be included in the eigenstates and energies using (degenerate) 
perturbation theory, and $H_{\text {eff }}$ evaluated as above. This gives expressions

$$
\begin{aligned}
J & =\frac{4}{27}\left[\frac{\left(2 t_{1}+t_{3}\right)^{2}\left(4 J_{H}+3 U\right)}{U^{2}}-\frac{8 J_{H}\left(9 t_{4}^{2}+2\left(t_{1}-t_{3}\right)^{2}\right)}{(2 U+3 \lambda)^{2}}\right] \\
K & =\frac{32 J_{H}}{9}\left[\frac{\left(t_{1}-t_{3}\right)^{2}-3\left(t_{2}^{2}-t_{4}^{2}\right)}{(2 U+3 \lambda)^{2}}\right] \\
\Gamma & =\frac{32 J_{H}}{9}\left[\frac{3 t_{4}^{2}+2 t_{2}\left(t_{1}-t_{3}\right)}{(2 U+3 \lambda)^{2}}\right] \\
\Gamma^{\prime} & =-\frac{32 J_{H}}{9}\left[\frac{t_{4}\left(t_{1}-t_{3}-3 t_{2}\right)}{(2 U+3 \lambda)^{2}}\right]
\end{aligned}
$$

up to corrections of order $O\left(J_{H}^{2} / \lambda^{2}\right)$ and $O\left(J_{H}^{2} / U^{2}\right)$. Note that the dependence of these exchanges on the hoppings is nearly identical in both perturbation theories.

\section{DERIVATION OF $\Gamma$ TERM}

To unambiguously show the existence of the $\Gamma$ term in the strong-coupling Hamiltonian, we derive the expression for $\Gamma$ explicitly. We will work in $U, J_{H} \gg \lambda \gg t$ limit to connect with the equations shown in the main text. The coefficient $\Gamma$ can then be determined from the matrix element

$$
\Gamma=2 i\left\langle-\frac{1}{2},-\frac{1}{2}\left|H_{\mathrm{eff}}\right|+\frac{1}{2}+\frac{1}{2}\right\rangle
$$

where $H_{\text {eff }}$ is the two-site effective Hamiltonian for a $x y(z)$ bond. To simplify notation we use the particle-hole mapping $d^{5} \rightarrow d^{1}$, mapping the Hamiltonian of Eq. 5 to

$$
H_{0}=H_{1}+H_{2}=\frac{U-3 J_{H}}{2}\left[\left(N_{1}-1\right)^{2}+\left(N_{2}-1\right)^{2}\right]-2 J_{H}\left(S_{1}^{2}+S_{2}^{2}\right)-\frac{J_{H}}{2}\left(L_{1}^{2}+L_{2}^{2}\right)
$$

Since particle-hole conjugation effectively maps $\left|+\frac{1}{2}\right\rangle \leftrightarrow\left|-\frac{1}{2}\right\rangle$ we see that we are interested in $\left\langle+\frac{1}{2},+\frac{1}{2}\left|H_{\mathrm{eff}}\right|-\frac{1}{2}-\frac{1}{2}\right\rangle=\left\langle-\frac{1}{2},-\frac{1}{2}\left|H_{\mathrm{eff}}\right|+\frac{1}{2}+\frac{1}{2}\right\rangle^{*}$ in this new basis. The eigenstates of $H_{1}$ or $H_{2}$ are eigenstates of $N, L^{2}, L_{z}, S^{2}$ and $S_{z}$ at each site, which we will denote as sets using terms symbols ${ }^{2 S+1} L$. Each individual eigenstates will be denoted as $\left|{ }^{2 S+1} L, M_{L}, M_{S}\right\rangle_{a}$ with the total number $N$ understood from context and $a=1,2$ denoting the site index. The ground state is an $N=1$ six-fold degenerate ${ }^{2} P$ state with energy $-5 J_{H} / 2$. Since the perturbation $T$ moves an electron from site 2 to site 1 we need then only consider $N_{1}=2$ and $N_{2}=0$ states. While the $N=0$ state is a trivial ${ }^{1} S$ state, anti-symmetric $N=2$ states can be formed by ${ }^{1} S,{ }^{3} P$ and ${ }^{1} D$ terms giving three relevant 
excitation energies

$$
\begin{aligned}
& E_{2}\left({ }^{1} S\right)+E_{0}\left({ }^{1} S\right)-2 E_{1}\left({ }^{2} P\right)=U+2 J_{H} \\
& E_{2}\left({ }^{3} P\right)+E_{0}\left({ }^{1} S\right)-2 E_{1}\left({ }^{2} P\right)=U-3 J_{H} \\
& E_{2}\left({ }^{1} D\right)+E_{0}\left({ }^{1} S\right)-2 E_{1}\left({ }^{2} P\right)=U-J_{H}
\end{aligned}
$$

where we have denoted the energy of the $N$-electron term ${ }^{2 S+1} L$ as $E_{N}\left({ }^{2 S+1} L\right)$. The most straightforward way to compute $\Gamma$ is to first decompose $T\left|+\frac{1}{2}\right\rangle_{1}\left|+\frac{1}{2}\right\rangle_{2}$ into eigenstates of $H_{1}+H_{2}$. Break $T$ into two parts $T=T_{1}+T_{2}$ with

$$
\begin{aligned}
& T_{1}=\sum_{\sigma}\left[t_{1}\left(d_{1, x z, \sigma}^{\dagger} d_{2, x z, \sigma}+d_{1, y z, \sigma}^{\dagger} d_{2, y z, \sigma}\right)+t_{3} \sum_{\sigma} d_{1, x y, \sigma}^{\dagger} d_{2, x y, \sigma}\right] \\
& T_{2}=t_{2} \sum_{\sigma}\left(d_{1, x z, \sigma}^{\dagger} d_{2, y z, \sigma}+d_{1, y z, \sigma}^{\dagger} d_{2, x z, \sigma}\right)
\end{aligned}
$$

Recall the definition of the $j_{\text {eff }}=1 / 2$ states as

$$
\begin{aligned}
\left|+\frac{1}{2}\right\rangle_{a} & =\sqrt{\frac{1}{3}}\left(d_{a, y z, \downarrow}^{\dagger}+i d_{a, x z, \downarrow}^{\dagger}+d_{a, x y, \uparrow}^{\dagger}\right)|0\rangle_{a}=\left[\sqrt{\frac{2}{3}} d_{a,+, \downarrow}^{\dagger}-i \sqrt{\frac{1}{3}} d_{a, 0, \uparrow}^{\dagger}\right]|0\rangle_{a} \\
\left|-\frac{1}{2}\right\rangle_{a} & =\sqrt{\frac{1}{3}}\left(d_{a, y z, \uparrow}^{\dagger}-i d_{a, x z, \uparrow}^{\dagger}-d_{a, x y, \downarrow}^{\dagger}\right)|0\rangle_{a}=\left[\sqrt{\frac{2}{3}} d_{a,-, \uparrow}^{\dagger}+i \sqrt{\frac{1}{3}} d_{a, 0, \downarrow}^{\dagger}\right]|0\rangle_{a}
\end{aligned}
$$

where $a=1,2$ and we have defined the $l_{\text {eff }}=1$ operators

$$
\begin{aligned}
& d_{a,+, \sigma}^{\dagger}=\sqrt{\frac{1}{2}}\left(d_{a, y z, \sigma}^{\dagger}+i d_{a, x z, \sigma}^{\dagger}\right) \\
& d_{a, 0, \sigma}^{\dagger}=i d_{a, x y, \sigma}^{\dagger} \\
& d_{a,-, \sigma}^{\dagger}=\sqrt{\frac{1}{2}}\left(d_{a, y z, \sigma}^{\dagger}-i d_{a, x z, \sigma}^{\dagger}\right)
\end{aligned}
$$

In this form we can now act each part of $T$, decomposing into the excited states as we go

$$
\begin{aligned}
T_{1}\left|+\frac{1}{2}\right\rangle_{1}\left|+\frac{1}{2}\right\rangle_{2} & =\left(\frac{t_{1}-t_{3}}{3}\right)\left(d_{1, y z, \downarrow}^{\dagger}+i d_{1, x z, \downarrow}^{\dagger}\right) d_{1, x y, \uparrow}^{\dagger}|0\rangle_{1}|0\rangle_{2} \\
& =-i\left(\frac{t_{1}-t_{3}}{3}\right)\left[|+\downarrow, 0 \uparrow\rangle_{1}-|0 \uparrow,+\downarrow\rangle_{1}\right]|0\rangle_{2} \\
& =-i\left(\frac{t_{1}-t_{3}}{3}\right)\left[\left|{ }^{3} P,+1,0\right\rangle_{1}-\left|{ }^{1} D,+1,0\right\rangle_{1}\right]|0\rangle_{2} \\
T_{2}\left|+\frac{1}{2}\right\rangle_{1}\left|+\frac{1}{2}\right\rangle_{2} & =\frac{t_{2}}{3}\left[2 d_{1, x z, \downarrow}^{\dagger} d_{2, y z, \downarrow}^{\dagger}+i\left(d_{1, y z, \downarrow}^{\dagger}-i d_{1, x z, \downarrow}^{\dagger}\right) d_{1, x y, \uparrow}^{\dagger}\right]|0\rangle_{1}|0\rangle_{2} \\
& =\frac{t_{2}}{3}[-2 i(|+\downarrow,-\downarrow\rangle-|-\downarrow,+\downarrow\rangle)+(|-\downarrow, 0 \uparrow\rangle-|0, \uparrow,-\downarrow\rangle)]|0\rangle_{2} \\
& \left.=\frac{t_{2}}{3}\left[-\left.2 \sqrt{2} i\right|^{3} P, 0,-1\right\rangle-\left|{ }^{3} P,-1,0\right\rangle-\left|{ }^{1} D,-1,0\right\rangle\right]|0\rangle_{2}
\end{aligned}
$$


To compute the matrix element we also need $T\left|-\frac{1}{2}\right\rangle_{1}\left|-\frac{1}{2}\right\rangle_{2}$ related to the states shown in Eq. 16 by the action of time-reversal

$$
\begin{aligned}
& \left.T_{1}\left|-\frac{1}{2}\right\rangle_{1}\left|-\frac{1}{2}\right\rangle_{2}=-i\left(\frac{t_{1}-t_{3}}{3}\right)\left[{ }^{3} P,-1,0\right\rangle_{1}-\left|{ }^{1} D,-1,0\right\rangle_{1}\right]|0\rangle_{2} \\
& \left.T_{2}\left|-\frac{1}{2}\right\rangle_{1}\left|-\frac{1}{2}\right\rangle_{2}=\frac{t_{2}}{3}\left[-2 \sqrt{2} i||^{3} P, 0,+1\right\rangle+\left|{ }^{3} P,+1,0\right\rangle+\left|{ }^{1} D,+1,0\right\rangle\right]|0\rangle_{2}
\end{aligned}
$$

where we have used that the $\left.\left.\right|^{2 S+1} L, M_{L}, M_{S}\right\rangle$ states pick up an additional sign $(-1)^{L+S+M_{L}+M_{S}}$ under time-reversal. In this form we can simply read off the matrix elements

$$
\begin{aligned}
\left\langle-\frac{1}{2},-\frac{1}{2}\left|H_{\mathrm{eff}}\right|+\frac{1}{2}+\frac{1}{2}\right\rangle & =4 i\left(\frac{t_{2}\left(t_{1}-t_{3}\right)}{9}\right)\left[\frac{1}{U-3 J_{H}}-\frac{1}{U-J_{H}}\right] \\
& =\frac{8 i J_{H}}{9}\left[\frac{t_{2}\left(t_{1}-t_{3}\right)}{\left(U-3 J_{H}\right)\left(U-J_{H}\right)}\right]
\end{aligned}
$$

Thus

$$
\Gamma=\frac{16 J_{H}}{9}\left[\frac{t_{2}\left(t_{1}-t_{3}\right)}{\left(U-3 J_{H}\right)\left(U-J_{H}\right)}\right]
$$

consistent with the expressions in the Eq. 5 of the main text and with Eq. $8 \mathrm{c}$ when $t_{4}=0$. 